\title{
Research Paper \\ Adaptive Coping Strategies in Patients With Spinal Cord Injury: A Phenomenological Study
}

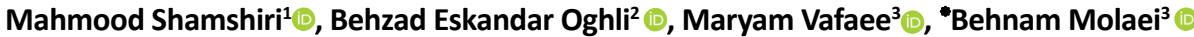

1. Department of Critical Care Nursing, Faculty of Nursing and Midwifery, Ardabil University of Medical Sciences, Ardabil, Iran. 2. Department of Surgery, Faculty of Medicine and Paramedical Sciences, Ardabil University of Medical Sciences, Ardabil, Iran. 3. Department of Psychiatry, Faculty of Medicine and Paramedical Sciences, Ardabil University of Medical Sciences, Ardabil, Iran.

\begin{tabular}{l|l}
$\begin{array}{l}\text { Use vour device to scan and } \\
\text { read the article online }\end{array}$ \\
Injury: A Phenomenological Study (Persian)]. Iranian Journal of Psychiatry and Clinical Psychology. 2021; 26(4):478-489. http:// \\
dx.doi.org/10.32598/ijpcp.26.4.2975.1 \\
dolinttp://dx.doi.org/10.32598/ijpcp.26.4.2975.1
\end{tabular}

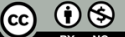

Received: 15 Jan 2020 Accepted: 13 Jul 2020 Available Online: 01 Jan 2021

Keywords: Spinal cord injures, Psychological adaptation, Life experience

\section{A B STRACT}

Objectives Spinal Cord Injury (SCI) is one of the most common causes of disability worlwide. Perception of coping strategies is important in these patients. This study aims to investigate the coping strategies in patients with $\mathrm{SCl}$.

Methods This is a hermeneutic phenomenological study using van Mann's approach. participants werre 15 adults (4 females, 11 males) with SCI living in Ardail, Iran who were recruited using a purposive sampling method. Semi-structured interviews were used to collect data. Interviews were recoreded and then transcribed and finally analyzed by using the thematic analysis approach proposed by van Mann. Results The lived experiences related to coping strategies in patients were summarized in six main themes including acceptence of the incurability, desire for being independent, praying for divine help, patience \& persuasion, time as a coping factor, and marriage.

Conclusion Patients with $\mathrm{SCl}$ use their own strategies to cope with their disability. Therefore, it is necessary for clinicians and psychiatrists to strengthen their coping strategies to help them better adapt to their injury.

\section{Extended Abstract}

\section{Introduction}

pinal Cord Injury (SCI) is one of the most traumatic events worldwide that affects all aspects of the patient's life. According to the World Health Organization, 250000-500000 people suffer a SCI each year, and its prevalence is estimated to be $40-80$ cases per million population. There is no comprehensive statistics for its prevalence in Iran, but according to one report, the annual incidence of $\mathrm{SCI}$ in Tehran is 44 cases per million population, and it is estimated that there are more than 70,000 patients with SCI in Iran. The most common average age at the time of SCI is $16-30$ years, followed by ages $21-45$ years and $46-60$ years.

One of the important health aspects of patients with $\mathrm{SCI}$ is their psychological dimension. Between 20 and $45 \%$ of SCI patients suffer depression. Suicide rates are 4-5 times higher in people with SCI. Other psychological problems associated with SCI include substance abuse, divorce, anger, damaged self-image, and behavior disorders. Adaptation mechanisms are a series of acquired strategies that can help patients to cope with life and health-related problems. Coping refers to any kind of behavioral or cognitive adjustments that people make for managing their health and life stressors. This strategy can vary depending on the type of stress and the

* Corresponding Author:

Behnam Molaei, PhD.

Address: Department of Psychiatry, Faculty of Medicine and Paramedical Sciences, Ardabil University of Medical Sciences, Ardabil, Iran.

Tel: +98 (45) 33726085

E-mail: b.molaei@arums.ac.ir 
cultural context of the individual. The present study aims to evaluate the adaptive coping strategies of patients with SCI.

\section{Methods}

This study is a hermeneutic phenomenological study conducted based on van Mann's approach in six steps: 1. Turning to lived experience; 2 . Investigating experience as we live it rather than as we conceptualize it; 3 . Reflecting on the essential themes which characterize the phenomenon; 4. Describing the phenomenon in the art of writing and rewriting; 5. Maintaining a strong and oriented relation to the phenomenon; and 6. Balancing the research context by considering parts and the whole. Purposive sampling method was used to recruit 15 people with SCI living in Ardabil, Iran whose primary language was Azeri. Semi-structured and in-depth interview of patients was conducted after obtaining permission from the Ethics Committee of Ardabil University of Medical Sciences and a written consent from the participants. Some asked questions were related to talking about daily life, how subjects feel about their problems, and how they cope with their problem. Interviews were recorded and transcribed. Then, data were analyzed using the thematic analysis guided by van Mann including detailed, selective and holistic techniques.

\section{Results}

The mean age of participants was 53.13 years. Of the 15 participants, 4 were female and 11 were male. In terms of marital status, one was single and 14 were married (Table 1). Exploring the lived experiences of the patients related to coping strategies revealed six main themes including: accepting the incurability, desire for being independent, praying for divine help, patience \& persuasion, time as a coping factor, and marriage.

\section{Discussion}

This study, which aimed to explore the coping strategies of people with SCI, revealed that they use effective coping strategies such as accepting the incurability, having desire for being independent, praying for divine help, being patient and persuasive, considering time as a coping factor, and marriage. This study indicated that the patients' understanding of the incurability of SCI is very important for

Table 1. Socio-demographic characteristics of the participants

\begin{tabular}{|c|c|c|c|c|c|c|}
\hline Participant No. & Age & Sex & Marital Status & Living With & Cause of Spinal Cord Injury & Level of Education \\
\hline 1 & 51 & M & Married & Spouse and children & War trauma & MSc. \\
\hline 2 & 62 & M & Married & Spouse and children & War trauma & Guidance school \\
\hline 3 & 70 & M & Married & Spouse and children & War trauma & Primary school \\
\hline 4 & 54 & M & Married & Spouse and children & War trauma & MSc. \\
\hline 5 & 54 & M & Married & Spouse and children & War trauma & Illiterate \\
\hline 6 & 27 & $\mathrm{~F}$ & Unmarried & Parents & Congenital & Primary school \\
\hline 7 & 23 & $\mathrm{~F}$ & Married & Spouse and child & Car accident & Primary school \\
\hline 8 & 65 & $\mathrm{~F}$ & Married & Spouse and children & Fall & Illiterate \\
\hline 9 & 56 & $M$ & Married & Spouse & Fall & Primary school \\
\hline 10 & 65 & $\mathrm{~F}$ & Married & Spouse & Car accident & Illiterate \\
\hline 11 & 42 & $M$ & Married & Spouse and child & Fall & High school \\
\hline 12 & 53 & $M$ & Married & Spouse & Tumor & Illiterate \\
\hline 13 & 63 & $M$ & Married & Spouse & Fall & Illiterate \\
\hline 14 & 51 & M & Married & Spouse and child & Car accident & Primary school \\
\hline 15 & 51 & M & Married & Spouse and children & War trauma & Illiterate \\
\hline
\end{tabular}


coping with loss, although it may lead to depression but it can help them accept it and continue to cope with the injury.

The desire for independence can play a very important role in better self-care and self-efficacy in patients with SCI. Other studies also have reported that patients with SCI have a substantial tendency towards self-reliance and independence and try to maintain their independence in daily life activities as much as possible. One of other effective coping strategies found in SCI patients was praying to God and his prophets for help. Therefore, divine help, religious beliefs and spirituality play a vital role in adapting to chronic conditions. A study by Wilson et al. (2017) reported that spirituality was strongly associated with the quality of life of SCI patients, and strengthening of their religious beliefs can improve their quality of life and better adaptation [19].

One of the themes that reflected a coping strategy in SCI patients was patience \& persuasion. In fact, patients with SCI maintain their mental well-being and even improve it by being patient and persuasive. It can be considered as a defense mechanism. Although this finding is not well reflected in other studies, it can be argued that strengthening of patience can help patients with SCI better cope with the injury. The Holy Qur'an has also emphasized all kinds of patience, especially patience in the face of adversity, that is, resilience after traumatic events and incurable diseases. A study by Valikhani and Khormaei (2015) on the prediction of pain based on patience showed that psychological factors, especially religious/psychological variables such as patience, can play an important role in relieving the physical and emotional dimensions of pain [24].

Patients in our study reported the time as a fundamental factor in coping with SCI. Heidegger, the famous phenomenologist also indicated the time as an essential facet of human being. Wijesuriya et al. (2012) in a study on patients with SCI also indicated to the important role of passing time in coping with SCI [25].

Marriage was the other important coping strategy in SCI patients. Their experiences showed that family formation increases life expectancy and better adaptation to disability. A study by Silveira et al. (2018) on life satisfaction and health status in patients with SCI showed that marital status is one of the predictors of life satisfaction, and married patients had higher life satisfaction than single patients. This finding is consistent with our result [27]. A study by Chan et al. (2000) found that people who got married before having SCI were more depressed than those who got married after having SCI. Since the effects of SCI are long-lasting, it affects not only the patient but also his/her spouse. Therefore, it is necessary for rehabilitation specialists to pay attention to the different needs of couples and the different ways to help them [28].

People with SCI use a variety of adaptive strategies to cope with the injury including acceptence of incurability, having desire for being independent, praying for divine help, being patient and persuasive, considering time as a coping factor, and marriage. Man, as a physical, mental, psychological and social being, always tries to adapt to disability after injury. Therefore, clinicians, psychologists and psychiatrists need to strengthen the reported coping strategies in SCI patients for their treatment.

\section{Ethical Considerations}

\section{Compliance with ethical guidelines}

This study obtained its ethical approval from the Research Ethics Committee of Ardabil University of Medical Sciences (Code: IR.ARUMS.REC.1397.123). Participants were fully informed about the study objectives and methods, and were assured of the confidentiality of their information. Prior to study, a written consent form was signed by all participants.

\section{Funding}

The present study was extracted from the MA. thesis of Behnam Molaei at the Department of Psychiatry, Faculty of Medicine and Paramedical Sciences, Ardabil University of Medical Sciences, Ardabil. It was financially supported, as a research project, by the Deputy for Research of Ardabil University of Medical Sciences.

\section{Authors contributions}

Methodology and data analysis; Mahmood Shamshiri; Editing \& review, and data analysis: Behzad Eskandar Oghli; Initial draft preparation and study report: Maryam Vafaee; Psychology advising and data analysis: Behnam Molaei.

\section{Conflicts of interest}

The authors declare no conflict of interest.

\section{Acknowledgements}

We are thankful to the participants who shared their experiences with us. In addition, we acknowledge Ardabil University of Medical Sciences (ARUMS) for financial support of the research project. 


\title{
راهبردهاى تطابق سازنده در بيماران داراى ضايعه نخاعى: يك مطالعه بديدهشناسى هرمنوتيك
}

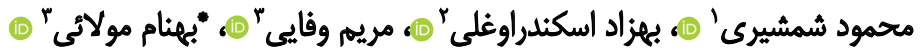 \\ ا. كروه يرستارى مراقبتهاى ويره، داتشكده يرستارى و مامايى، دانشكاه علوميزشكى اردبيل، اردبيل، ايران.

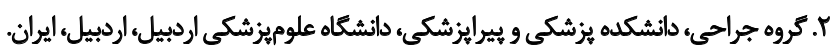

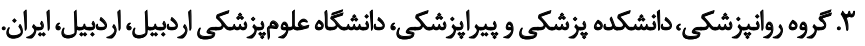

\begin{abstract}
حكيد

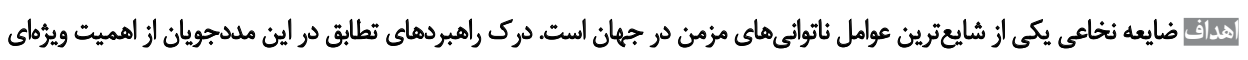

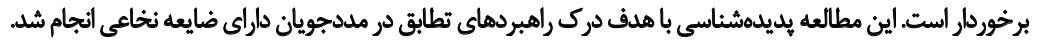

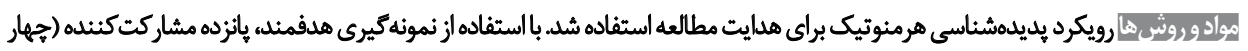

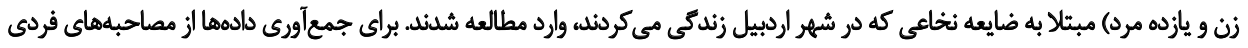

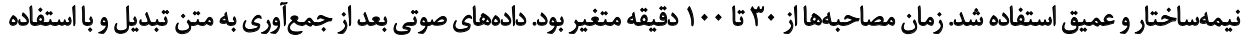

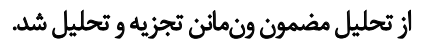

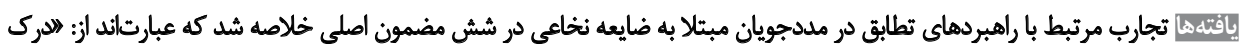

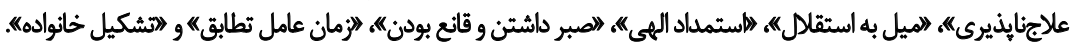

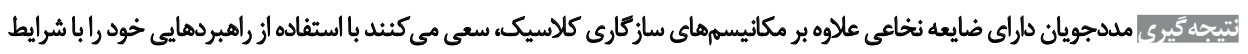

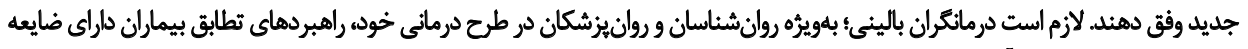

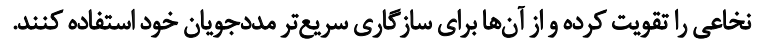

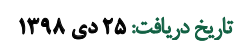

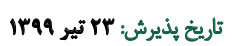

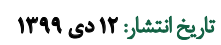

كليدوازوها:

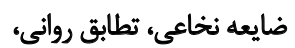

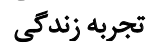

ضايعات نخاعى يكى از ناكوارترين مشكلاتي است كه مي تواند

dale

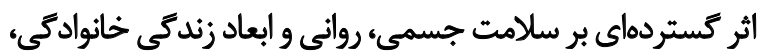

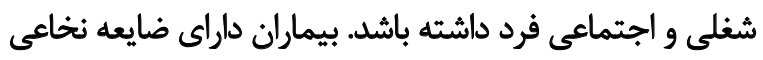

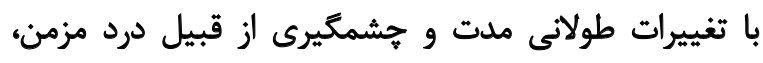

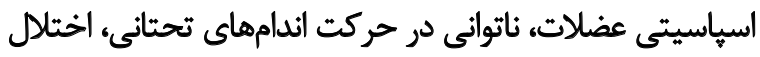

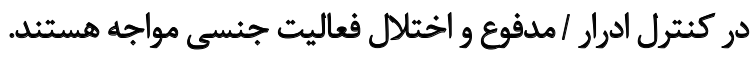

جوارض ناشى از اين آسيب مي تواند فرد را از هرخده اقتصادى

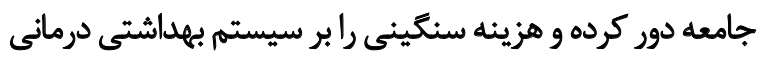

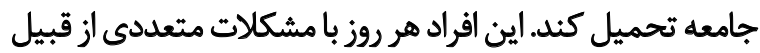

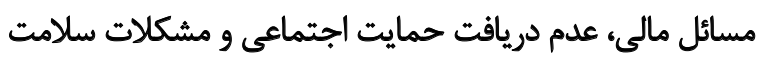

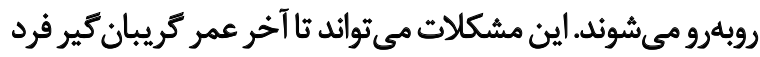

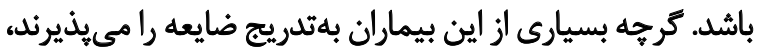

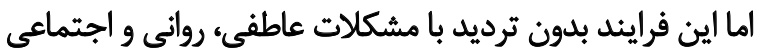

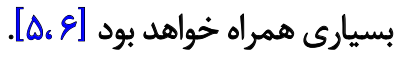

يكى از ابعاد مهم سلامت بيماران داراى ضايعه نخاعى، بُعد

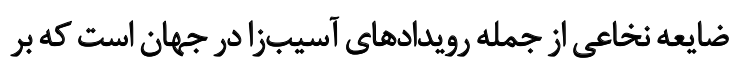

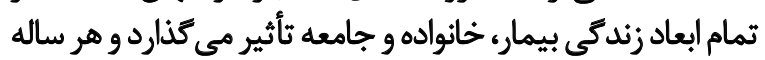

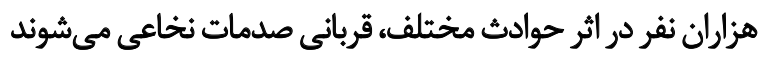

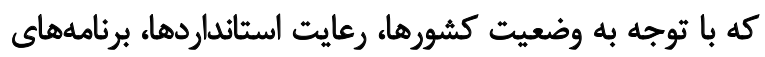

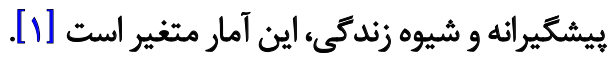

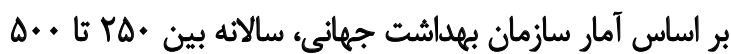

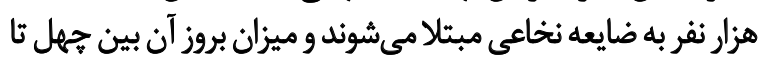

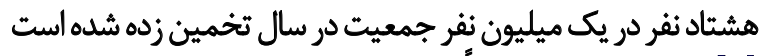

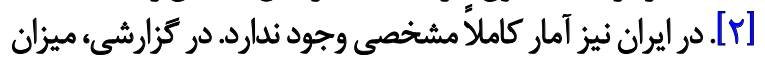

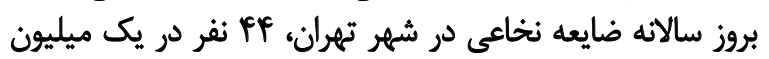

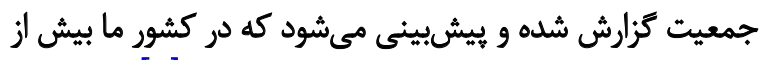

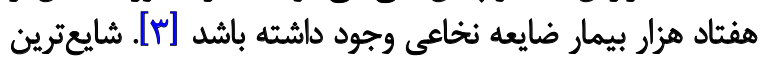

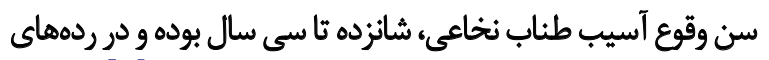

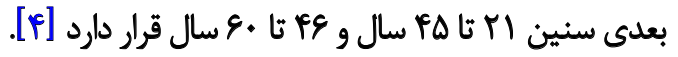

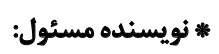

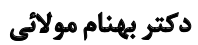

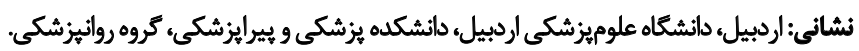

تلفن:

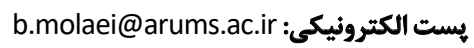


قوى و معطوف به يديده مورد مطالعه؛ و. ايجاد توازن در زميئه

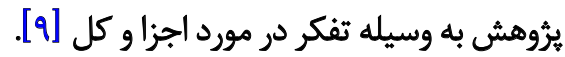

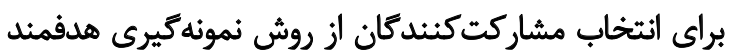

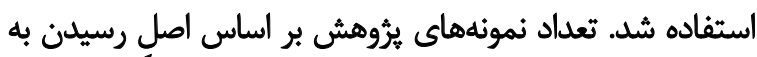

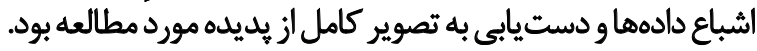

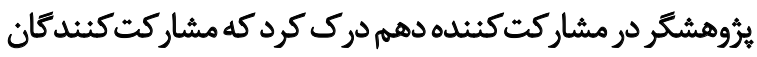

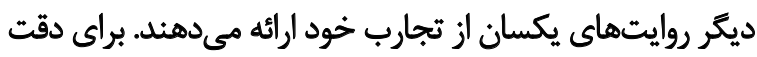

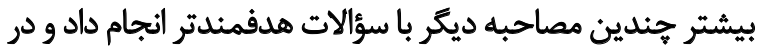

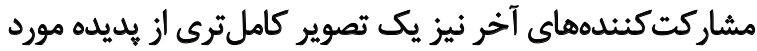

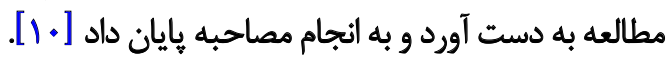
تحليل دادهها در مطالعات كيفى، يك فرايند جرخهاى است

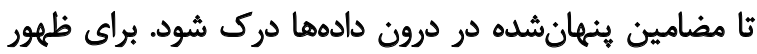

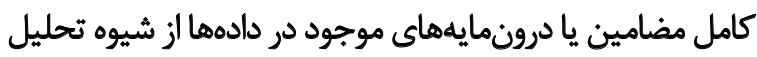

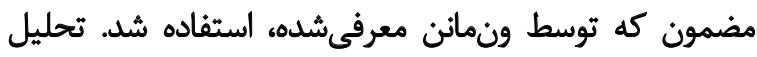

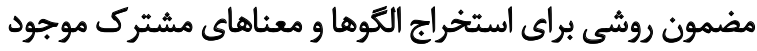

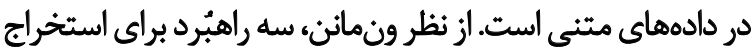

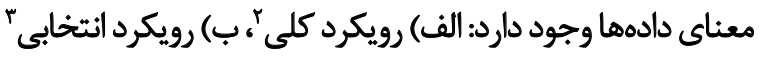
وج) رويكرد جزء به جزء

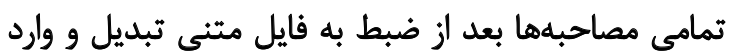

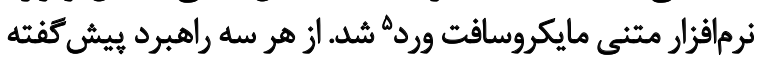

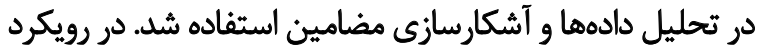

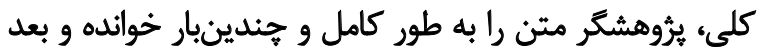

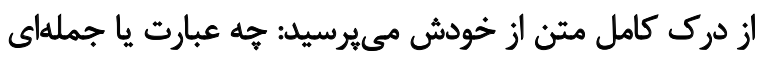

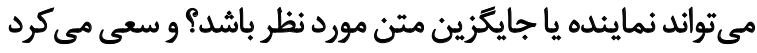

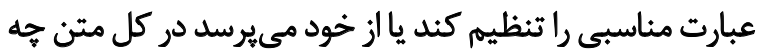

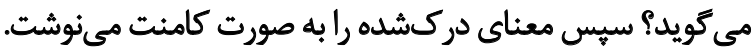

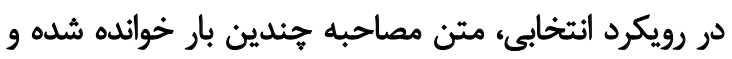

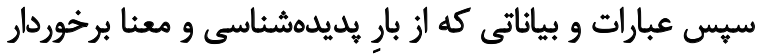

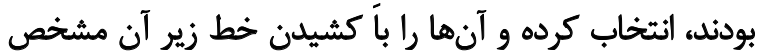

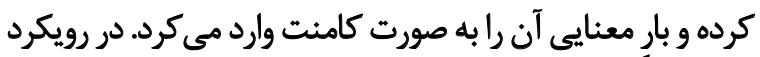

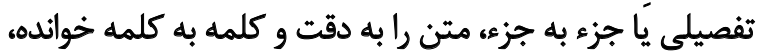

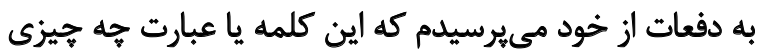

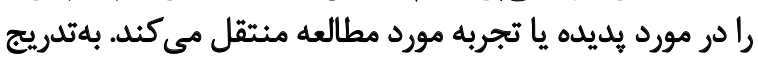

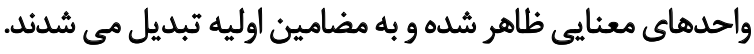

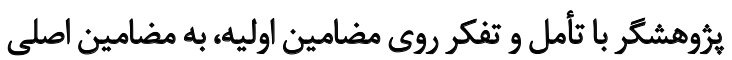

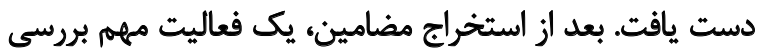

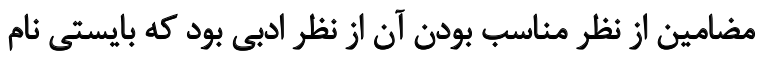

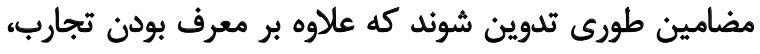

2. Holistic Approach

3. Selective Approach

4. Detailed Approach

5. Microsoft word

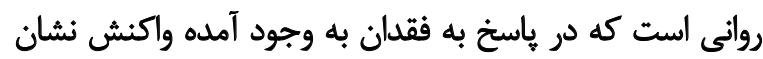

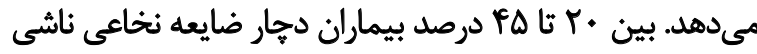

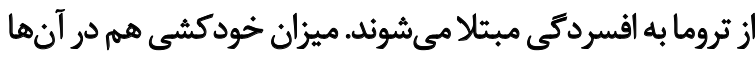

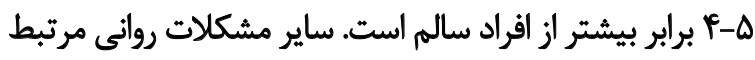

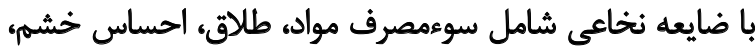
آسيب به هويت فردى و اختلال در كنترل رفتار است [Vائل]

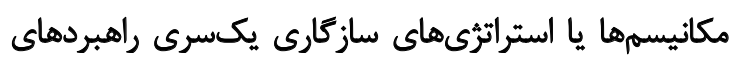

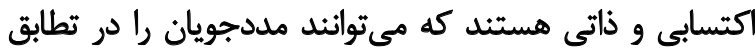

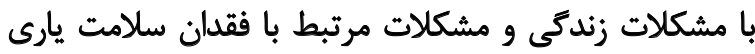

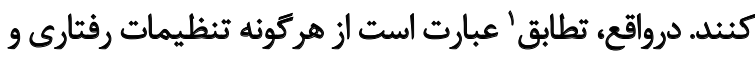

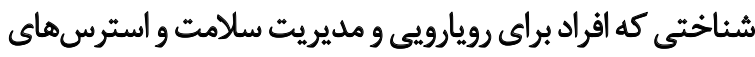

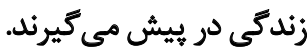

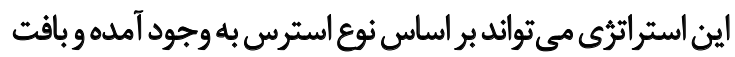

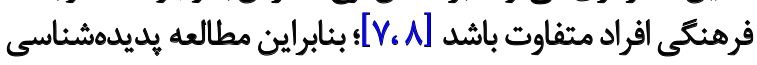

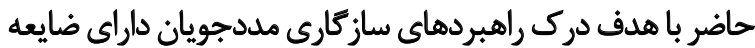
نخاعى آذرى زبان در شهر اردبيل انجام شدا ساري

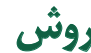

براى هدايت اين مطالعه از رويكرد بديدهشناسى هرمنوتيك

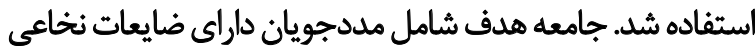

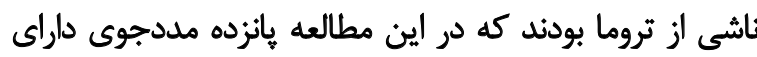

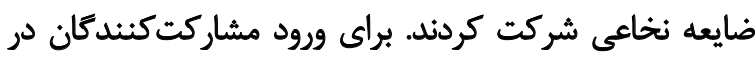

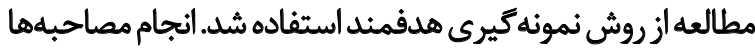

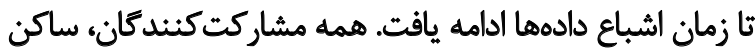

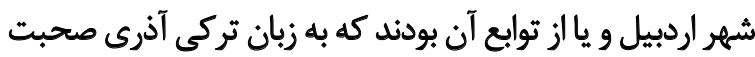

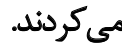

براى جمعآورى دادهها از مصاحبه نيمهساختار و عميق استفاده

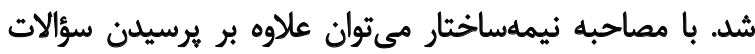

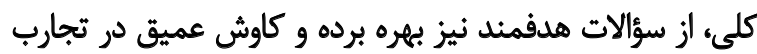

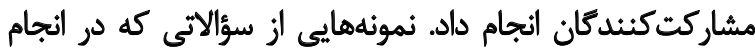

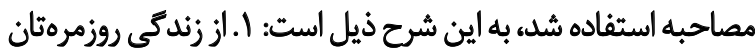

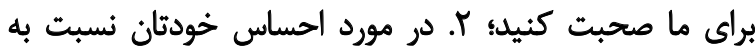
مشكلتان صحبت كنيد؛ "ا. جكونه با اين مشكل كنار آمديد؟ براى انجام مطالعه، در عمل از روش ونمانن استفاده شد كه

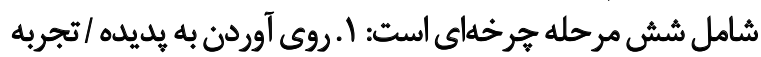

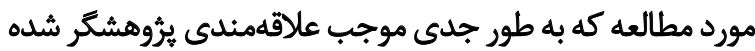

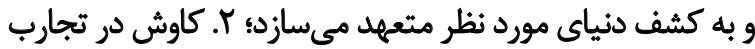

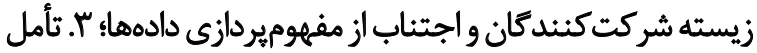

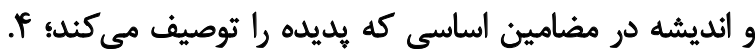
توصيف يديده از طريق هنر نوشتن و بازنويسى الهي هـ حفظ رابطه 
جدول ا. مشخصات جمعيتشناختى اجتماعى مشاركت كندكان

\begin{tabular}{|c|c|c|c|c|c|c|}
\hline تحصيلات & علت ضايعه نخاعى & ز زندىى با & وضعيت تأهل & جنس & سن & مشاركت كنده \\
\hline فوق ليسانس & جانباز & همسر و فرزئلان & متأهل & مذكر & $\Delta 1$ & 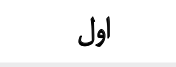 \\
\hline اول راهنمايع & جانباز & همسر و فرزندان & متأهل & مذكر & gr & دوم \\
\hline دوم ابتداييى & جاثباز & همسر و فرزندان & متأهل & مذكر & v. & سوم سوم \\
\hline فوق ليسانس & جاثباز & همسر وفرزندان & مثأهل & مذكر & $\Delta f$ & جهارم \\
\hline بـ بـواد & جانباز & همسر وفرزندان & مثاهل & مذكر & $\Delta f$ & ينجم \\
\hline ينجم ابثدايع & بيمارى مادرزادى & والدين & مجرد & مؤنث & $r r$ & ششم \\
\hline ينجم ابتدايى & تصادف & همسر و فرزند & متأهل & مؤنث & $r r$ & هفتم \\
\hline بيسواد & سقوط از ارتفاع & همسر و فرزندان & متأهل & مؤنث & sa & 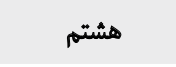 \\
\hline سوم ابتدايى & سقوط از ارتفاع & همسر & متأهل & مذكر & $\Delta$ & نهم \\
\hline بىسواد & تصادف & همسر & متأهل & مؤنث & $9 \Delta$ & دهم \\
\hline دوم دييرستان & سقوط از ارتفاع & همسر و فرزند & متأهل & مذكر & rr & يازدهم \\
\hline بىسواد & تومور نخاعى & همسر & متأهل & مذكر & $\Delta r$ & دوازدهم \\
\hline بي بواد & سقوط از ارتفاع & همسر & مثأهل & مذكر & gr & سيزدهم \\
\hline سوم ابتدايع & تصادف & همسر وفرزند & متأهل & مذكر & $\Delta 1$ & جهارتهم \\
\hline ل بىسواد & جانباز & همسر و فرزندان & متأهل & مذكر & Q) & ل بانزدهم \\
\hline
\end{tabular}

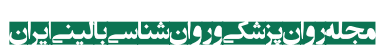

\section{مضمون اول: دوك علاجنائذيرى}

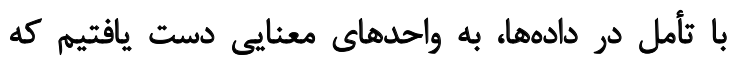

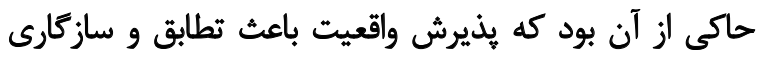

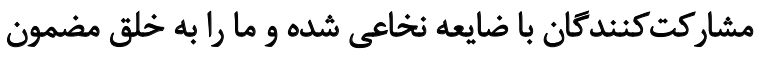

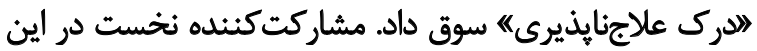

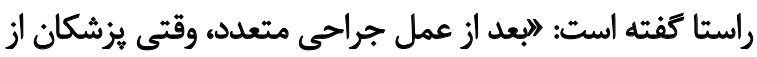

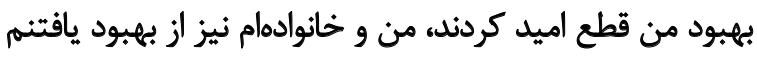

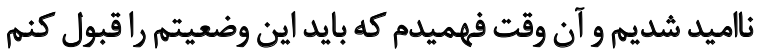

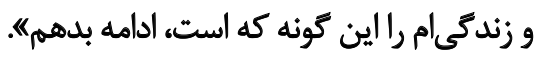

مشاركت كنئده ششم كفته است: لاوقتى اين اتفاق (ضايعه

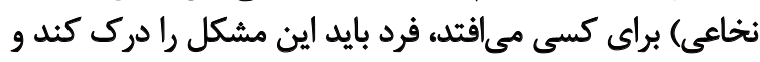

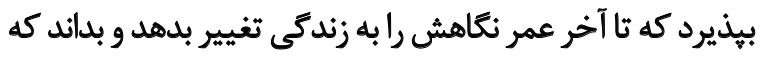

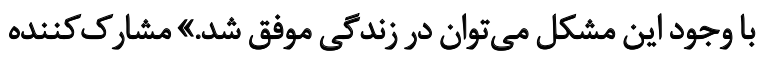

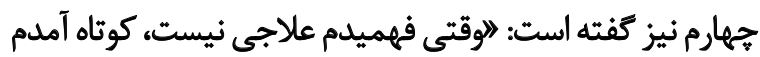

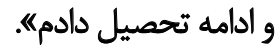

\section{مضمون دوم: ميل به استقالال}

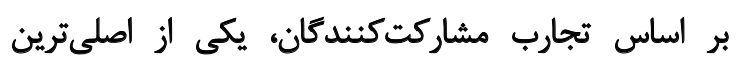
استراتزىهاى سازكار شدن با شرايط جديد در بيماركان بان ضايعه

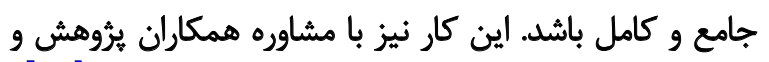

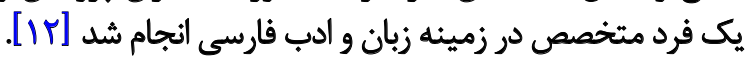
مراى روايى يا موثق بودن يافتهها در اين مطالعه از راهبردهاي

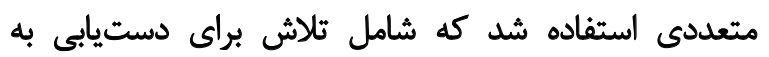

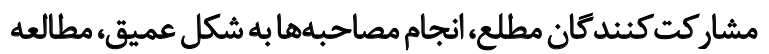

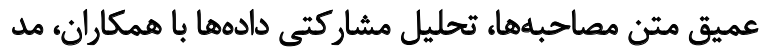

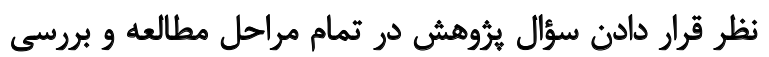

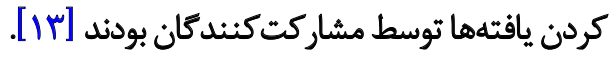

ياقتلهها

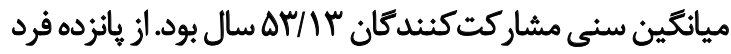

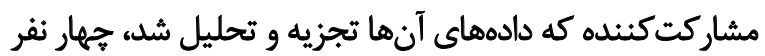

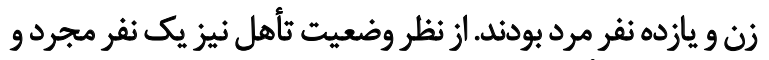

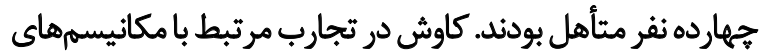

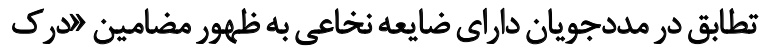

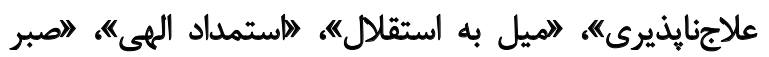

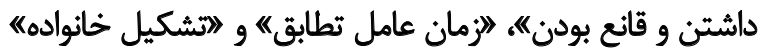

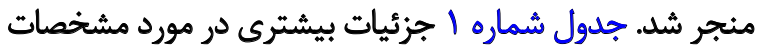

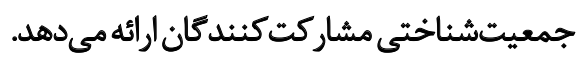


المن هم با قرآن خواندن، دعا كردن و نماز خواندن به قلبم آرامش

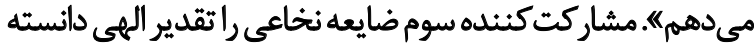

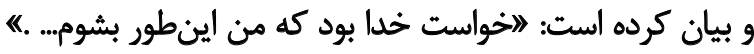
وى در جاى ديكر از مصاحبه اشاره كرده است: البه كمك و و ياد ياد

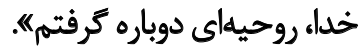

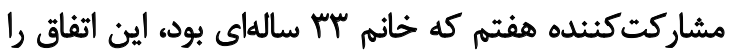

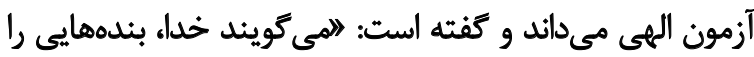

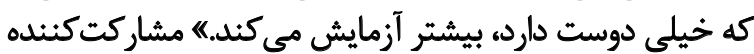

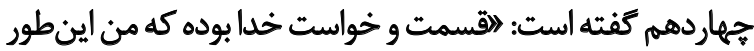

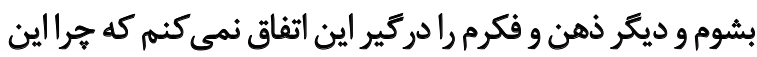

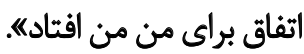

\section{مضمون حهمارم: صبر داشتن و قانع بودن}

صحبثهاى متعددى از مددجويان ضايعه نخاعى وجود دارد كه

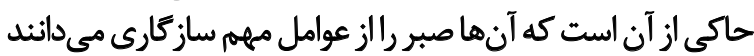

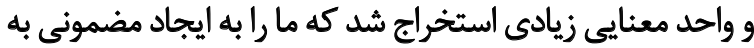

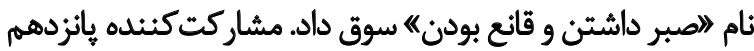

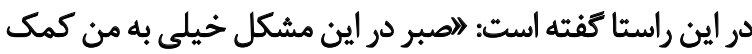

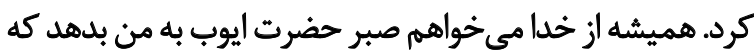

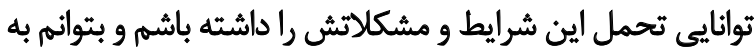

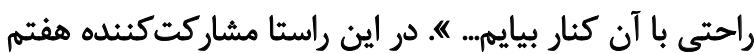

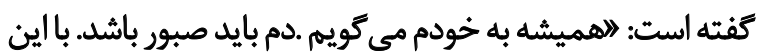

$$
\text { حرفها خودم را آرام و قانع مى كنم.... }
$$

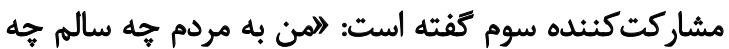

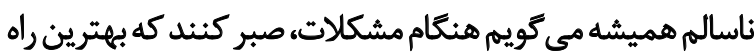

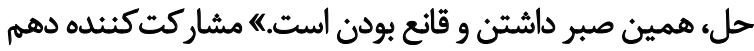

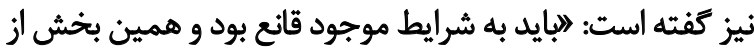

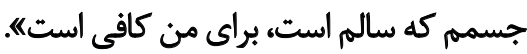

\section{مضمون يُجمه: زمان عامل تطابثي}

مصاحبههاى مشاركت كنندكان مملو از صحبتهايیى بود كه

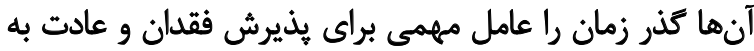

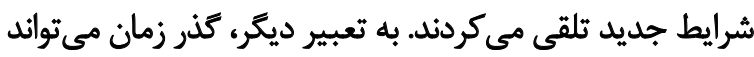

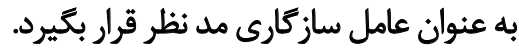

مشاركت كننده ششم كفته است: الاوايل، زندكى براى من من بن

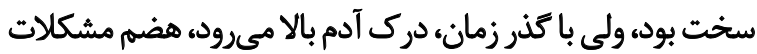

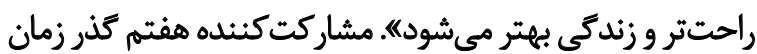

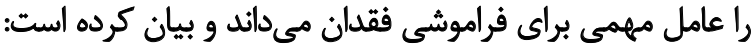

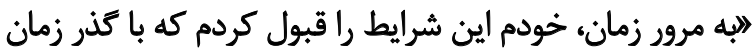

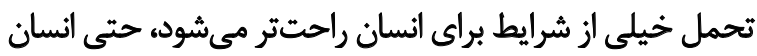

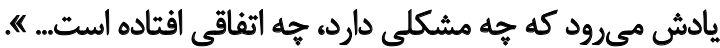
مشاركت كنئده جهارم كفته است: البه نظر من كذر زمان خيلى

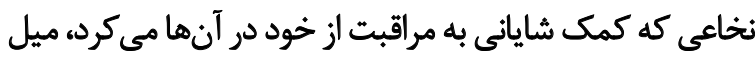

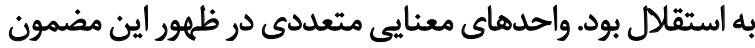

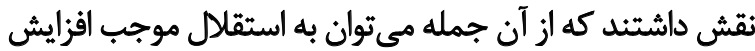

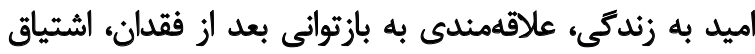

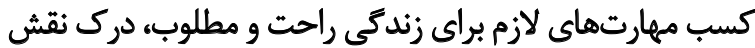

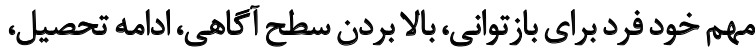
ورزش، هنر و روى آوردن به كار اشاره كرد. بردي

در واقع بخش مهمى از صحبتهاى مشاركتكنيندان

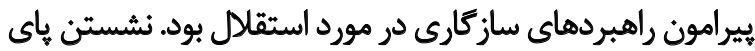

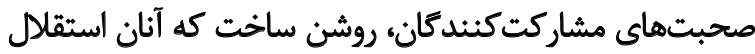

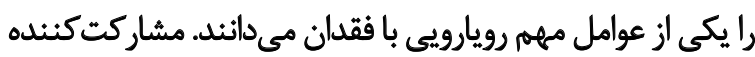

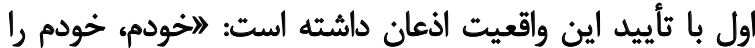

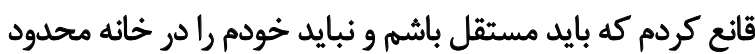

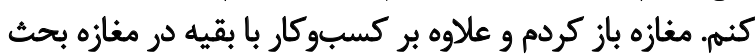

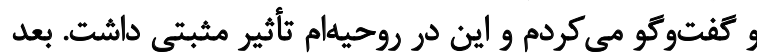

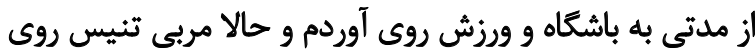

$$
\text { ميز هستمى مدتى باش. }
$$

مشاركتكنيده ينجم خود را عامل مهمى براي استقلال

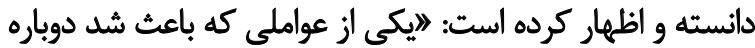

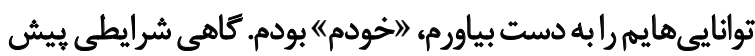

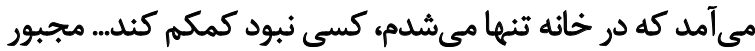

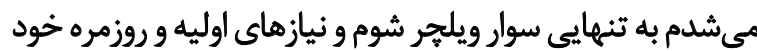

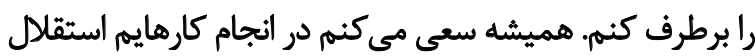

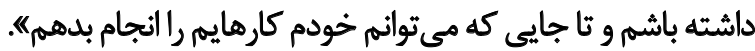

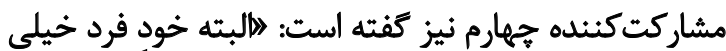

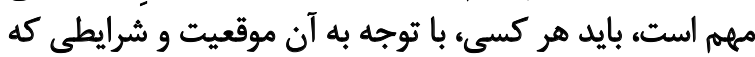

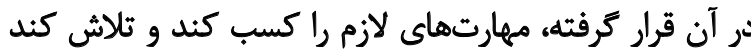

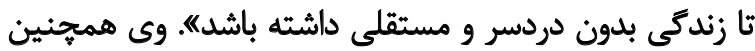

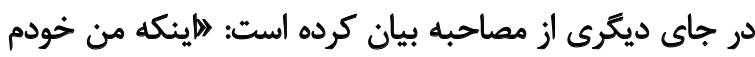

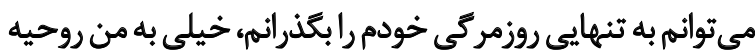
مي مي (1)

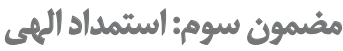
از ميان صحبتهاي مشاركت كنيدكان، تعداد زيادى واحدهاي

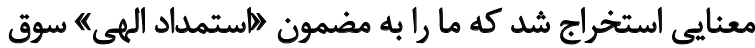

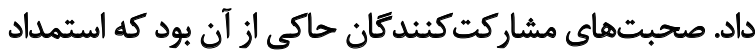

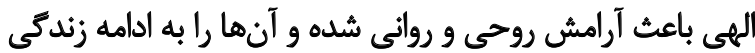

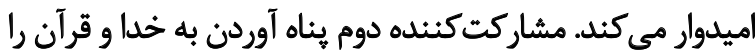

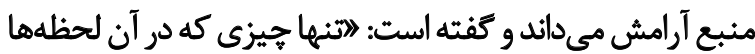

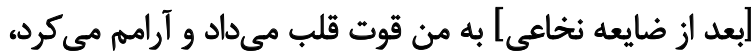
يناه آوردن به خدا و قرآن خواندن بوده مشاركت كننده هشتم نيز با تأييد اين مطلب بيان كرده است: 


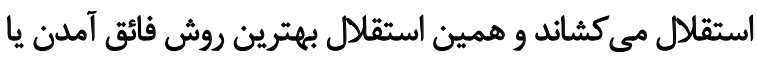

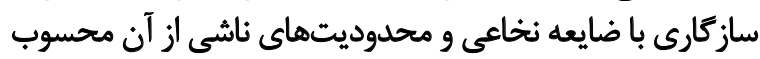

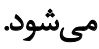

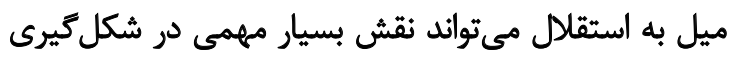

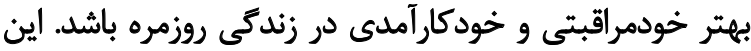
راهبرد رويارويى در مطالعات ديكران نيز به اشكال مختلف ديد ديده

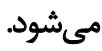

در مطالعهاى كزارش شده كه بيمار ان ضايعه نخاعى، تمايل ويرٔهاى

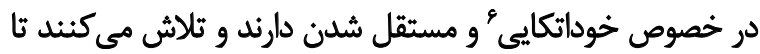

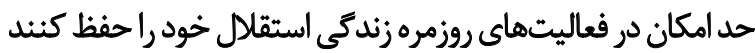

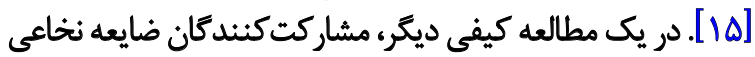

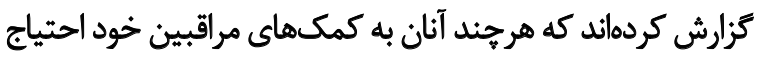

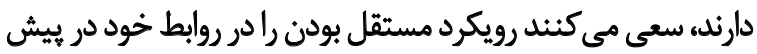

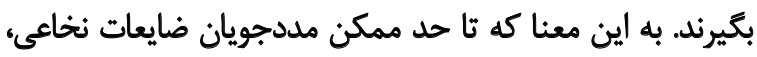

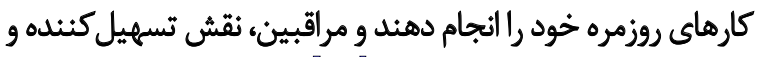

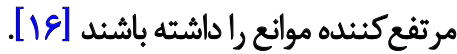

يكى ازمؤلفه هاى مرهمى كه در ايجاداستقلال مشاركت كنيندكان

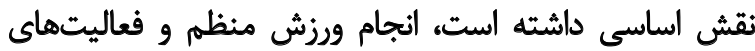

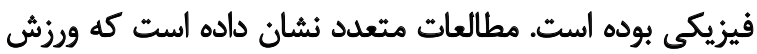

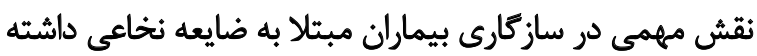

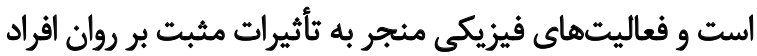

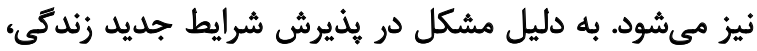

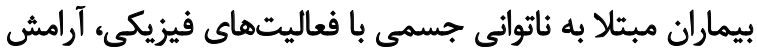
ذهنى بيدا مي كنيند.

ورزش در بيماران ضايعه نخاعى باعث بهبود حضور آنها در

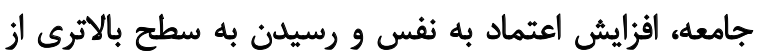

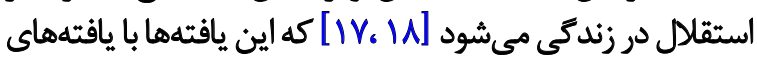
يُؤوش حاضر همخواني دارد.

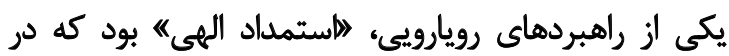

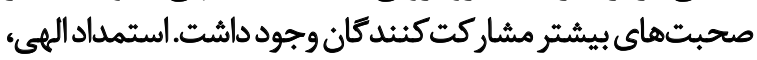

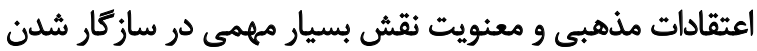

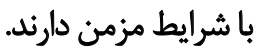

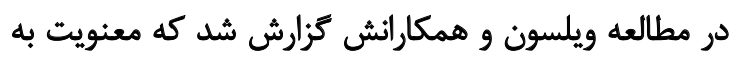

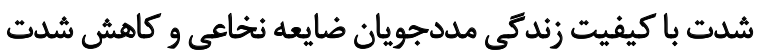

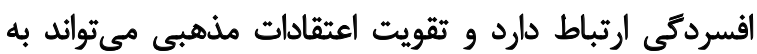

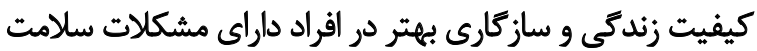

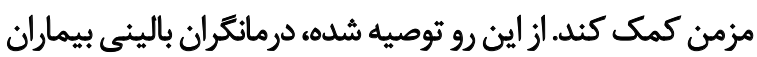
داراى آسيب نخاعى را از نظر معنويت ارزيابي كنئد [19] مطالعه ديكر نشان داد كه اضطراب و افسردگى دو عارضه مههم
ههم است، كذزر زمان خيلى از مشكلات را حل مي كثند، شايد

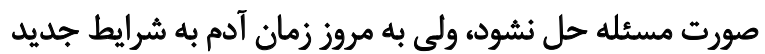

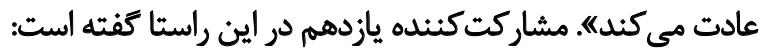

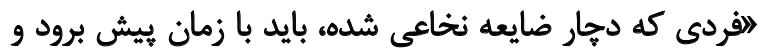

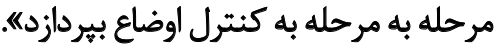
مضمون ششهم: تشكيل خانواده بخشى از تجارب مشاركت كنيدكان روشّن ساخت كه تشكيل

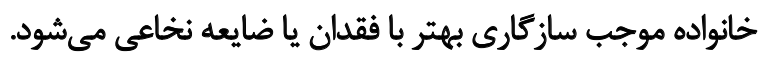

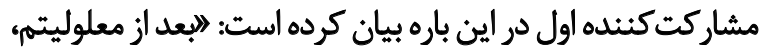

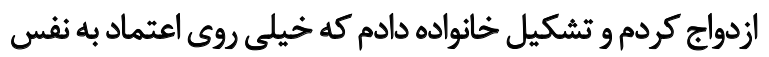

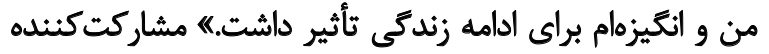

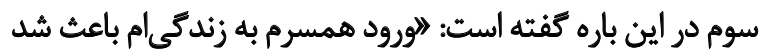

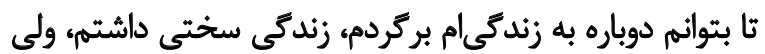

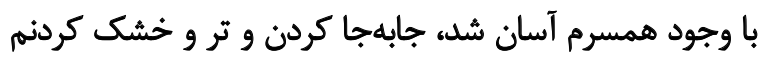

برعهده همسيرم:بود. هون

ث

اين مطالعه كه باهدف درى راهبردهاي ساز كارى در مددجويان

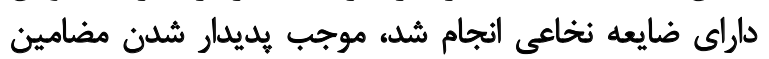

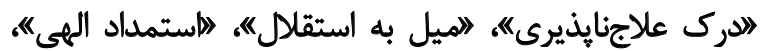

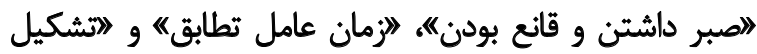

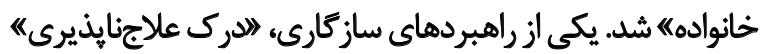
بود كه يك روش سازكاري شناختى مهمى به به حساب مي آيد.

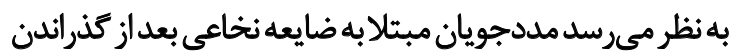

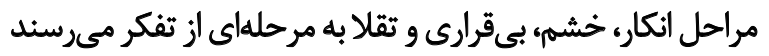

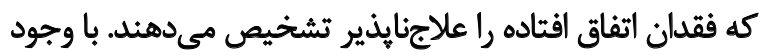

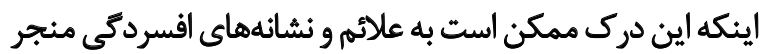

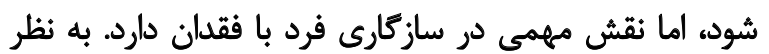

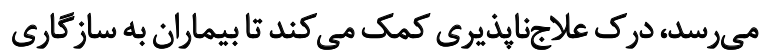
رسيده و دنياى خود را بازتعريف كنيند.

كسلر به ينج مرحله كلاسيك سوك يرداخته و آن رابه ترتيب

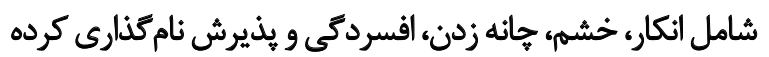

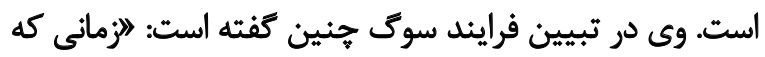

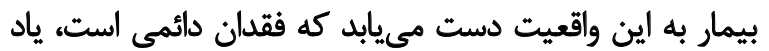

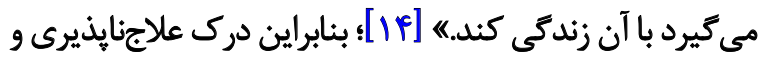

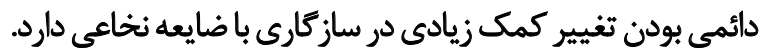
ميل به استقلال و مستقل بودن در امور زندكى روزمره در همه إندان

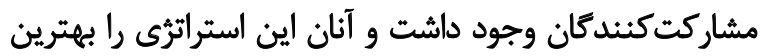

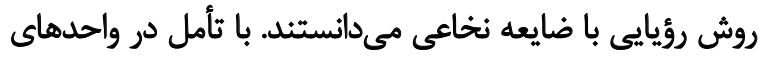

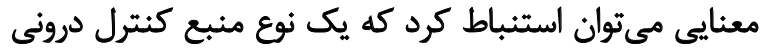

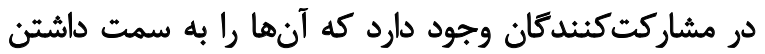




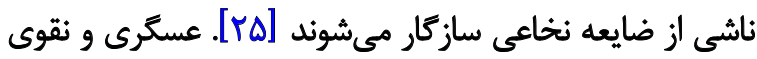

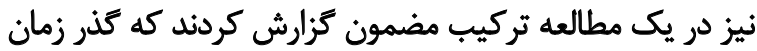

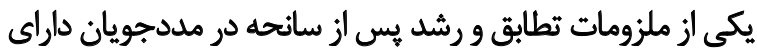

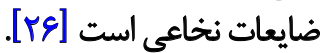

تشكيل خانواده نيز از جمله راهبردهاى سازكارى بود كه از

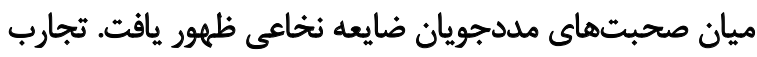

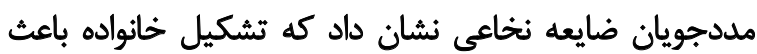

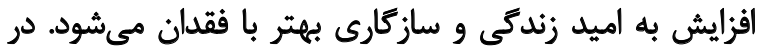

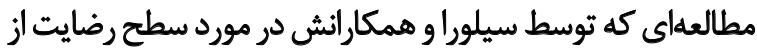

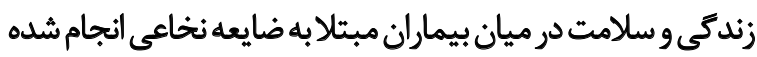

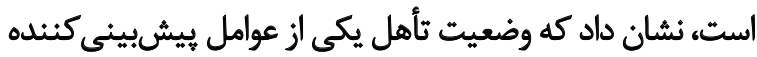

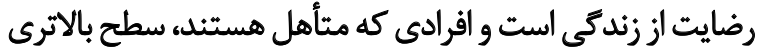

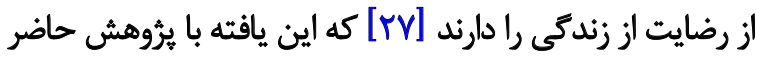

$$
\text { همخوانى دارد. }
$$

مطالعهاى كه توسط حِان و همكاران انجام شده نشان داد كه

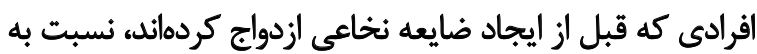

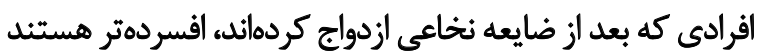

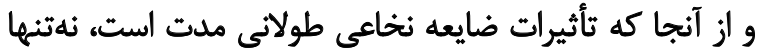

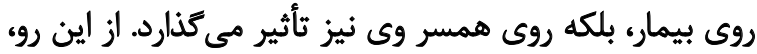

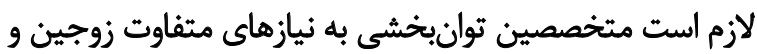

شيوههاى مختلف كمك به آنها توجه كنيند [YMA]

يثروهش حاضر محدوديتهايى داشت كه لازم است در تردير

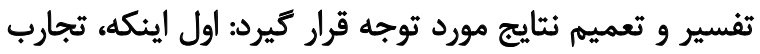

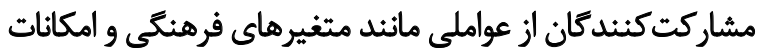

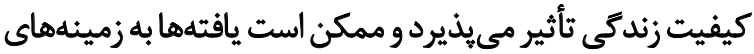

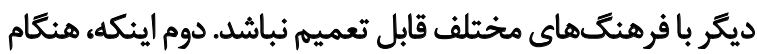

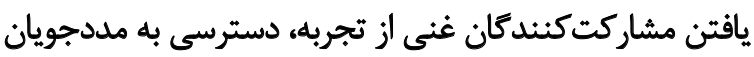

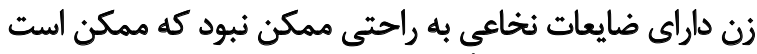

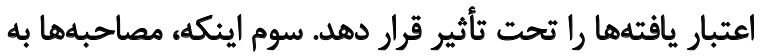

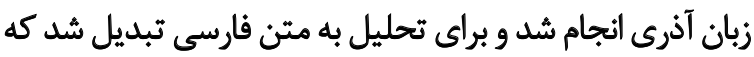

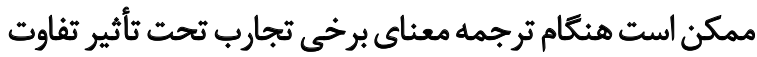

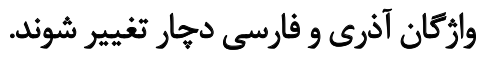

يشيشهاد مىشود در مطالعات آتى، يُوهشهائى مشابه با

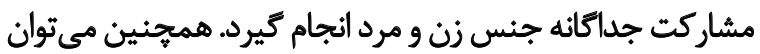

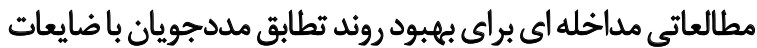

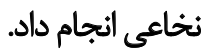

\section{نتيجلئيرى}

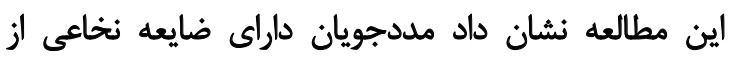

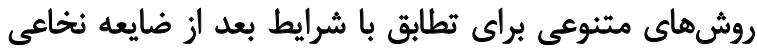

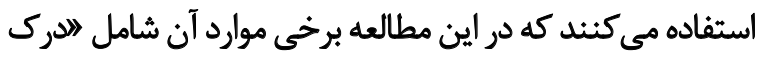

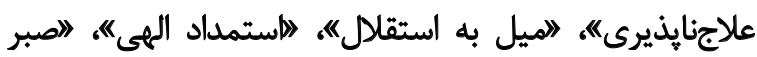

در مددجويان با ضايعه نخاعى است كه دين و اعثقادات مذهبيى

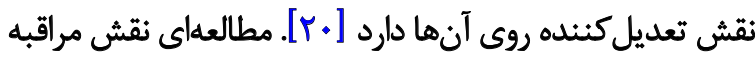

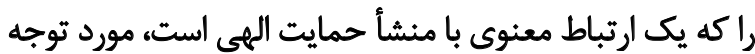

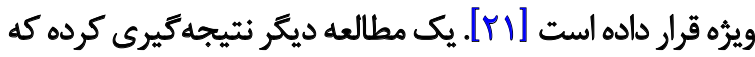

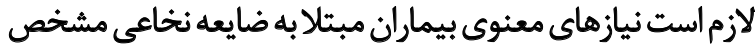

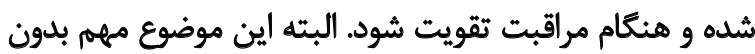

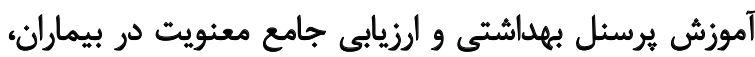
موفقيت آميز نخواهد بود [Tr.

ابراهيمى و همكاران نيز در يك مطالعه توصيفى كزارش كردند

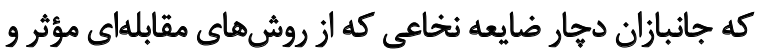

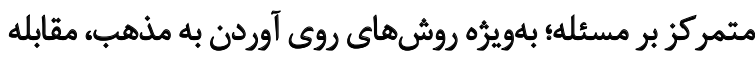

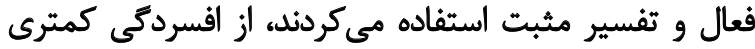

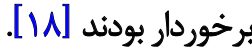

يكى از مضمونهاى تشكيل دهنده راهبردهاى رويارويى الصبر

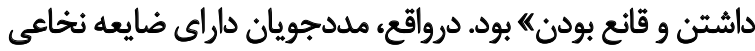

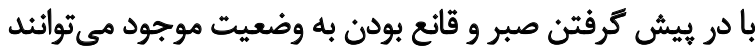

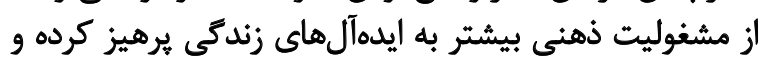

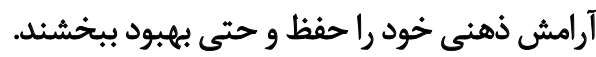

مى توان صبر داشتن و قانع بودن را يك مكانيسم دفاعى بخته

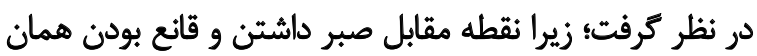

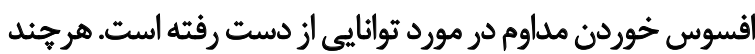

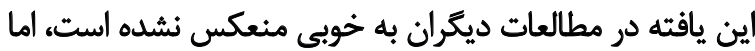

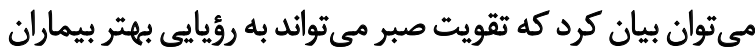

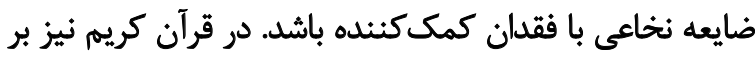

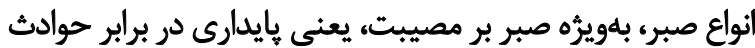
تلخ و ناكوار و بيمارى لاعلاج تأكيد شده است [بريت.

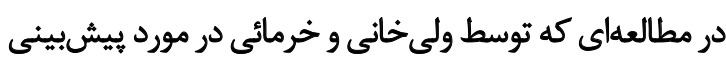

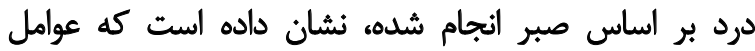

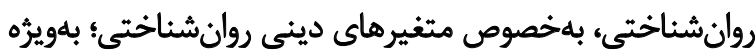

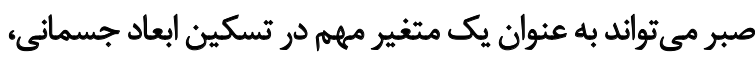

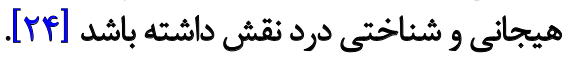

"ازمان عامل تطابقه يكى از مضمونهايى بود كه از ميان

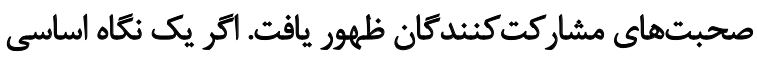

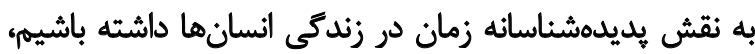

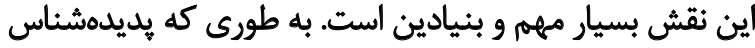

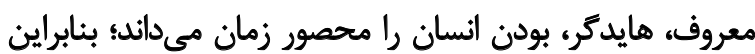

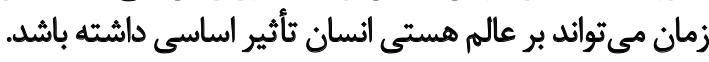

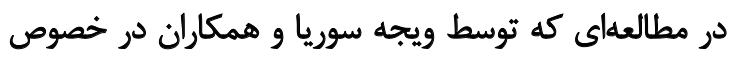

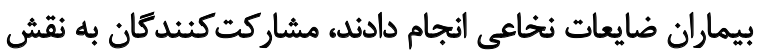

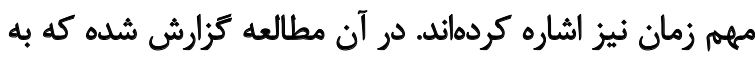
مرور زمان تعداد بيشترى از بيماران ضايعات نخاعى بان فقدان فئان 
داشتن و قانع بودنه، "زمان عامل تطابق" و "تشكيل خانواده《) مشخص شد.

بنابراين مىتوان نتيجهيرى كرد كه انسان به عنوان يك

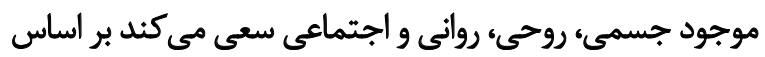

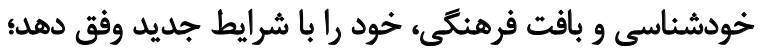

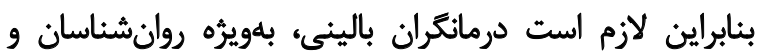

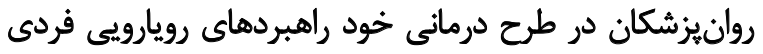

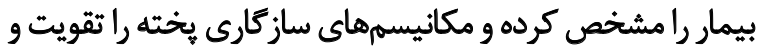
از آنها براى تطابق مؤثرتر مددجويان خود استفاده كنيند.

مالاحظات اخلاقى

\section{بيروى از اصول اخلاق يثوهش}

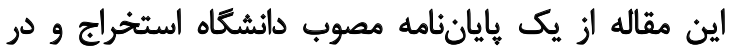

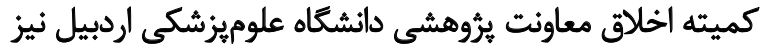

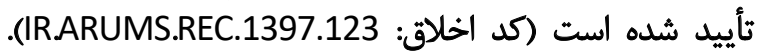

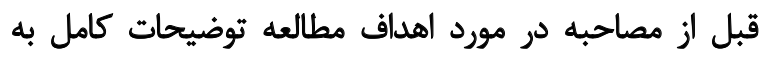

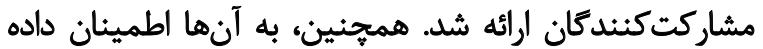

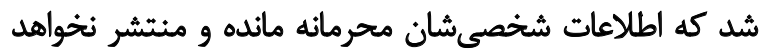

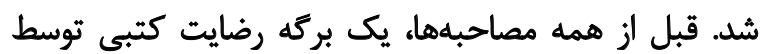
مشاركت كنند مان مطالعه و امضاشد.

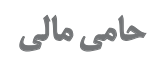

اين مقاله از يايان نامه كارشناسى نويسنده آخر، در كروه

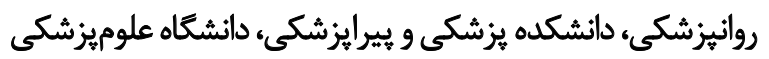

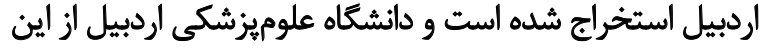
تحقيق حمايت مالى كرده است.

مشار كت نويسئد مكان

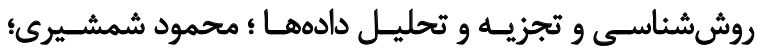

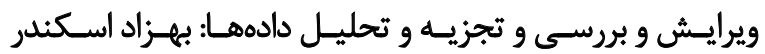

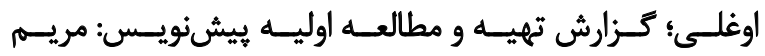

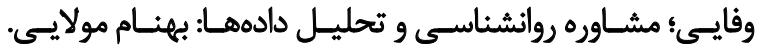

$$
\text { تعارض منافع }
$$

بنابر اظهار نويسندكان، اين مقاله تعارض منافع ندارد.

$$
\text { تشكر وقدردائي }
$$

از دانشكاه علوميزشكى اردبيل به جهت حمايت مالى طرح

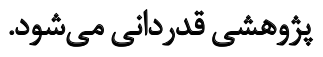




\section{References}

[1] van den Berg ME, Castellote JM, Mahillo-Fernandez I, de Pedro-Cuesta J. Incidence of spinal cord injury worldwide: A systematic review. Neuroepidemiology. 2010; 34(3):18492. [DOI: 10.1159/000279335]. [DOI:10.1159/000279335] [PMID]

[2] World Health Organization. Spinal Cord Injury. Geneva: World Health Organization; 2013. https:/ /www.who.int/ news-room/fact-sheets/detail/spinal-cord-injury

[3] Rahimi-Movaghar V, Saadat S, Rasouli M, Ganji S, Ghahramani $M$, Zarei $M$, et al. Prevalence of spinal cord injury in Tehran, Iran. The Journal of Spinal Cord Medicine. 2009; 32(4):428-31. [DOI:10.1080/10790268.2009.11754572] [PMID] [PMCID]

[4] Chen Y, Tang Y, Vogel L, DeVivo M. Causes of spinal cord injury. Topics in Spinal Cord Injury Rehabilitation. 2013; 19(1):1-8.[DOI:10.1310/sci1901-1] [PMID] [PMCID]

[5] Fekete C, Wahrendorf M, Reinhardt JD, Post MW, Siegrist J. Work stress and quality of life in persons with disabilities from four European countries: The case of spinal cord injury. Quality of Life Research. 2014; 23(5):1661-71. [DOI:10.1007/s11136-013-0610-7] [PMID]

[6] Paul C, Derrett S, McAllister S, Herbison P, Beaver C, Sullivan M. Socioeconomic outcomes following spinal cord injury and the role of no-fault compensation: Longitudinal study. Spinal Cord. 2013; 51(12):919-25. [DOI:10.1038/ sc.2013.110] [PMID]

[7] Arya S, Xue S, Embuldeniya A, Narammalage H, da Silva $\mathrm{T}$, Williams $\mathrm{S}$, et al. Coping strategies used by traumatic spinal cord injury patients in Sri Lanka: A focus group study. Disability and Rehabilitation. 2016; 38(20):2008-15. [DOI:10. 3109/09638288.2015.1111433] [PMID]

[8] Peláez-Ballestas I, Boonen A, Vázquez-Mellado J, ReyesLagunes I, Hernández-Garduño A, Goycochea MV, et al. Coping strategies for health and daily-life stressors in patients with rheumatoid arthritis, ankylosing spondylitis, and gout: STROBE-compliant article. Medicine. 2015; 94(10):e600. [DOI:10.1097/MD.0000000000000600] [PMID] [PMCID]

[9] Moghadam ZB, Ghiyasvandian S, Shahbazzadegan S, Shamshiri M. Parenting experiences of mothers who are blind in Iran: A hermeneutic phenomenological study. Journal of Visual Impairment and Blindness. 2017; 111(2):11322. [DOI:10.1177/0145482X1711100203]

[10] Creswell JW, Poth CN. Qualitative Inquiry and Research Design: Choosing Among Five Approaches. Essex: Vicki Knight; 2018. https://books.google.com/books?id=Ykrux or10cYC\&printsec $=$ frontcover\&dq $=$

[11] Shamshiri M, Mohammadi N, Cheraghi MA, Vehvilainen-Julkunen K, Sadeghi T. Disciplined care for disciplined patients: Experience of hospitalized blind patients. Holistic Nursing Practice. 2013; 27(6):344-8. [DOI:10.1097/ HNP.0b013e3182a72bcd] [PMID]

[12] Van Manen M. Researching lived experience: Human science for an action sensitive pedagogy. Milton Park: Routledge; 2016. https://books.google.com/books?id=1LZmD AAAQBAJ\&printsec $=$ frontcover\&dq $=$
[13] Pereira HR. Rigour in phenomenological research: Reflections of a novice nurse researcher. Nursing Research. 2012; 19(3):16-9. [DOI:10.7748/nr2012.04.19.3.16.c9054] [PMID]

[14] Kessler D. Finding Meaning: The sixth stage of grief. New York: Scribner; 2019. https:/ / books.google.com/book $\mathrm{s} ? \mathrm{id}=\mathrm{H} 920 \mathrm{DwAAQBAJ} \&$ printsec $=$ frontcover\&dq=

[15] Nikbakht-Nasrabadi A, Mohammadi N, Yazdanshenas M, Shabany M. Toward overcoming physical disability in spinal cord injury: A qualitative inquiry of the experiences of injured individuals and their families. BMC Neurology. 2019; 19(1):171. [DOI:10.1186/s12883-019-1391-6] [PMID] [PMCID]

[16] Jeyathevan G, Cameron JI, Craven BC, Munce SEP, Jaglal SB. Re-building relationships after a spinal cord injury: Experiences of family caregivers and care recipients. BMC Neurology. 2019; 19(1):117. [DOI:10.1186/s12883-019 1347-x] [PMID] [PMCID]

[17] Krakowska N, Szmelcer BP, Zaborna D, Fortuna A Wszelaki P, Florczak A, et al. Rehabilitation of patients with paraplegia-a review of the diversity of forms. Journal of Education, Health and Sport. 2019; 9(5):534-46. https:/ / www. researchgate.net/publication/345627447

[18] Ebrahimi A, Bolhari J, Zolfaghari F. [Stress coping strategies and social support in depressive veterans with spinal cord injury (Persian)]. Iranian Journal of Psychiatry and Clinical Psychology. 2002; 8(2):40-8. http://ijpcp.iums. ac.ir/article-1-235-en.html

[19] Wilson CS, Forchheimer M, Heinemann AW, Warren AM, McCullumsmith C. Assessment of the relationship of spiritual well-being to depression and quality of life for persons with spinal cord injury.Disability and Rehabilitation. 2017; 39(5):491-6. [DOI:10.3109/09638288.2016.115260 0] [PMID]

[20] Rahnama P, Javidan AN, Saberi H, Montazeri A, Tavakkoli S, Pakpour AH, et al. Does religious coping and spirituality have a moderating role on depression and anxiety in patients with spinal cord injury? A study from Iran. Spinal Cord. 2015; 53(12):870-4. [DOI:10.1038/sc.2015.102] [PMID]

[21] Littooij E, Widdershoven GAM, Stolwijk-Swüste JM, Doodeman S, Leget CJW, Dekker J. Global meaning in people with spinal cord injury: Content and changes. The Journal of Spinal Cord Medicine. 2016; 39(2):197-205. [DOI:10.1179 /2045772314Y.0000000290] [PMID] [PMCID]

[22] Jones KF, Dorsett P, Briggs L, Simpson GK. The role of spirituality in Spinal Cord Injury (SCI) rehabilitation: exploring health professional perspectives. Spinal Cord Ser Cases. 2018; 4:54. [DOI:10.1038/s41394-018-0078-3] [PMID] [PMCID]

[23] Amani R, Ebadi Z, Rezaei S. Study of Patience Domains in Quran. Quranic Knowledge Research. 2016; 7(25):133-56. [DOI: 10.22054/rjqk.2016.7088]

[24] Valikhani A, Khormaei F. Religion and pain: prediction of pain dimensions based on patience. Iran Journal of Nursing. 2015; 28(95):1-10. [DOI:10.29252/ijn.28.95.1]

[25] Wijesuriya N, Tran Y, Middleton J, Craig A. Impact of fatigue on the health-related quality of life in persons with spinal cord injury. Arch Phys Med Rehabil. 2012; 93(2):31924. [DOI:10.1016/j.apmr.2011.09.008] [PMID] 
[26] Asgari Z, Naghavi A. Explaining post-traumatic growth: Thematic synthesis of qualitative research. Iranian Journal of Psychiatry and Clinical Psychology. 2019; 25(2):222-34. [DOI:10.32598/ijpcp.25.2.222]

[27] Silveira SL, Ledoux TA, Johnston CA, Kalpakjian C, O'Connor DP, Cottingham M, et al. Well on wheels intervention: Satisfaction with life and health for adults with spinal cord injuries. The Journal Of Spinal Cord Medicine. 2018; 17 December: 1-9. [DOI:10.1080/10790268.2018.15543 33] [PMID] [PMCID]

[28] Chan RC, Lee PW, Lieh-Mak F. Coping with spinal cord injury: Personal and marital adjustment in the Hong Kong Chinese setting. Spinal Cord. 2000; 38(11):687-96. [DOI:10.1038/sj.sc.3101085] [PMID] 